\title{
Generalization of the results on the effect of selenium nanocomposites on the interaction of potatoes with the ring rot disease pathogen
}

\author{
Perfileva A.I.*, Nozhkina O.A., Graskova I.A. \\ Siberian Institute of Plant Physiology and Biochemistry, Siberian Branch, Russian Academy of Sciences, \\ Irkutsk, Russia \\ *email: alla.light@mail.ru
}

Selenium plays an important role in plants. It is present in a number of redox enzymes as a cofactor, takes part in the formation of chlorophyll, synthesis of tricarboxylic acids, in the metabolism of long-chain fatty acids. It has an antagonistic effect on the absorption and transport of heavy metals, increases resistance to water stress, salt and drought resistance. In addition, selenium is involved in the synthesis of tocopherols, tocotrienols, and ubiquinones. Direct transformations of selenium compounds in a plant cell occur in chloroplasts (synthesis of selenocysteine from selenite) and cytoplasm (synthesis of selenomethionine and selenocysteine). Many studies have been carried out to study the effect of nanocomposites of selenium on plant growth and development, as well as their ability to resist stresses of various nature.

We are studied the biological activity of nanocomposites of selenium in natural polymer matrices (carrageenan, arabinogalactan, starch). It was revealed that these agents reduce the viability of the phytopathogenic bacterium Clavibacter sepedonicus (reduces cell viability and their ability to form biofilms) and phytopathogenic fungi of the Phytophthora. Some of the studied chemically synthesized nanocomposites stimulate the growth and development of potatoes in vitro, the germination of seeds of cultivated plants (soybeans, peas, potatoes). Experiments in plants have shown a stimulating effect from nanocomposites on the plant biometric indicators and a decreased harmful effect from bacterial infection. Nanocomposites activate the antioxidant system of plant cells, affect the activity of peroxidase and products of lipid peroxidation. X-ray microanalysis was shown that after the treatment of plants with nanocomposites, selenium did not accumulate in plant tissues. In addition, chemically synthesized nanocomposites based on natural polymer matrices did not inhibit the viability of soil microorganisms Acinetobacter guillouiae, Rhodococcus erythropolis, Pseudomonas oryzihabitans, which indicates a relative safety for real use. These results allow us to consider nanocomposites as a plant development stimulator for agricultural crops. 